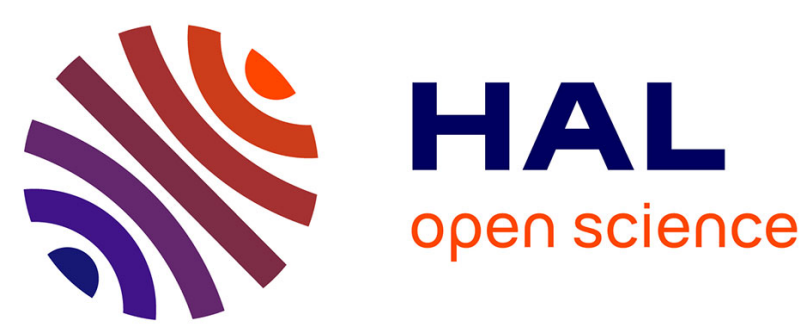

\title{
The tree to the left, the forest to the right: Political attitude and perceptual bias
}

Serge Caparos, Simon Fortier-St-Pierre, Jérémie Gosselin, Isabelle Blanchette, Benoit Brisson

\section{- To cite this version:}

Serge Caparos, Simon Fortier-St-Pierre, Jérémie Gosselin, Isabelle Blanchette, Benoit Brisson. The tree to the left, the forest to the right: Political attitude and perceptual bias. Cognition, 2015, 134, pp.155-164. 10.1016/j.cognition.2014.10.006 . hal-01275996

\section{HAL Id: hal-01275996 https://hal.science/hal-01275996}

Submitted on 1 Apr 2016

HAL is a multi-disciplinary open access archive for the deposit and dissemination of scientific research documents, whether they are published or not. The documents may come from teaching and research institutions in France or abroad, or from public or private research centers.
L'archive ouverte pluridisciplinaire HAL, est destinée au dépôt et à la diffusion de documents scientifiques de niveau recherche, publiés ou non, émanant des établissements d'enseignement et de recherche français ou étrangers, des laboratoires publics ou privés.

$$
\text { Copyright }
$$




\title{
The tree to the left, the forest to the right: Political attitude and perceptual bias
}

\author{
Serge Caparos ${ }^{1}$, Simon Fortier-St-Pierre², Jérémie Gosselin², Isabelle Blanchette ${ }^{2}$, Benoit Brisson ${ }^{2}$ \\ ${ }^{1}$ Université de Nîmes, CHROME EA 7352, Rue du Dr Georges Salan, 30000, Nîmes, France \\ ${ }^{2}$ Université du Québec à Trois-Rivières, 3351 boulevard des Forges, CP 500, Trois-Rivières, G9A \\ $5 \mathrm{H} 7$, Canada
}

\section{Contact:}

\section{serge.caparos@unimes.fr}

Key words: Political attitudes, Cognitive rigidity, Visual perception, Local/global bias, Navon, Ebbinghaus illusion

\section{Publication information}

Published in Cognition. Elsevier copyright. This article may not exactly replicate the final version published in the Elsevier journal. It is not the copy of record.

Published version of the article may be found at this link:

http://www.sciencedirect.com/science/article/pii/S0010027714002066

Article DOI:

10.1016/j.cognition.2014.10.006

(C)2015. This manuscript version is made available under the CC-BY-NC-ND 4.0 license http://creativecommons.org/licenses/by-nc-nd/4.0/ 


\begin{abstract}
A prominent model suggests that individuals to the right of the political spectrum are more cognitively rigid and less tolerant of ambiguity than individuals to the left. On the basis of this model, we predicted that a psychological mechanism linked to the resolution of visual ambiguity - perceptual bias - would be linked to political attitude. Perceptual bias causes western individuals to favour a global interpretation when scrutinizing ambiguous hierarchical displays (e.g., alignment of trees) that can be perceived either in terms of their local elements (e.g., several trees) or in terms of their global structure (e.g., a forest). Using three tasks (based on Navon-like hierarchical figures or on the Ebbinghaus illusion), we demonstrate (1) that rightoriented Westerners present a stronger bias towards global perception than left-oriented Westerners and (2) that this stronger bias is linked to higher cognitive rigidity. This study establishes for the first time that political ideology, a high-level construct, is directly reflected in low-level perception. Right- and left-oriented individuals actually see the world differently.
\end{abstract}


Political attitude and perceptual bias

\section{The tree to the left, the forest to the right: Political attitudes and perceptual bias}

\section{Introduction}

As a powerful force that can affect lives of millions of individuals (e.g., Luguri, Napier, \& Dovidio, 2012), political ideology is crucially important to human societies and it is essential to better understand its underpinnings and its correlates across neurocognitive functioning (e.g., Kanai, Feilden, Firth, \& Rees, 2011; Oxley et al., 2008; Smith, Oxley, Hibbing, Alford, \& Hibbing, 2011). In this line of research, one remarkable study (Amodio, Jost, Master, \& Yee, 2007) has revealed basic neurocognitive differences between left- and right-oriented individuals (also referred to, respectively, as liberals and conservatives). In a Go/No-Go task - in which participants give a response on frequent $(\mathrm{Go})$ trials and must withhold their response on rare (No-Go) trials - right-oriented individuals were less able to inhibit responses on rare trials than left-oriented individuals, and they showed a weaker neural response linked to conflict monitoring (error-related negativity; Young, Botvinick, \& Cohen, 2004). The authors argued that right-oriented individuals are prone to higher persistence and lower flexibility for rare-occurring events, compared to left-oriented individuals. These findings are consistent with a major psychological model of political attitudes holding that right-oriented individuals need higher cognitive closure: they are less tolerant of uncertainty and of ambiguity than left-oriented individuals; they are more cognitively rigid (Jost, Glaser, Kruglanski, \& Sulloway, 2003).

If the cognitive-rigidity view is true, psychological mechanisms linked to ambiguity resolution should be related to political attitude. While cognitive rigidity is generally understood as a high-level construct (e.g., rigid categorisation or judgments; Jost et al., 2003), it can potentially have correlates in low-level psychological processes. Specifically, cognitive rigidity might affect mechanisms involved in the resolution of perceptual ambiguity such as perceptual bias. When scrutinizing ambiguous hierarchical displays (e.g., alignment of trees) that can be perceived either in terms of their local elements (e.g., several trees) or in terms of their global structure (e.g., a forest), perception tends to be consistently biased towards one interpretation over the other (Navon, 1977; Davidoff, Fonteneau, \& Fagot, 2008). Extending the cognitiverigidity hypothesis (Jost et al., 2003), we might anticipate that perception could be more strongly biased in right-oriented individuals, because they would show a greater propensity to disambiguate equivocal visual information always in the same way. While studies have documented links between political attitudes and cognitive function such as inhibition, no study has yet examined possible correlates in terms of lower-level perceptual processes. Here we test the extent to which left and right-oriented individuals see the world differently.

If right-oriented Westerners indeed show stronger perceptual bias, in principle this could mean either a stronger local bias (i.e., the trees are prioritised most of the time) or a stronger global bias (i.e., the forest is prioritised most of the time). However, given that in western culture most individuals naturally show a global bias (local bias is rare; e.g., in Caparos et al., 2012 , only $13 \%$ of the Western participants showed a preference for local figures), a parsimonious prediction is that right-oriented Westerners should show a stronger global bias. 
This idea is supported by findings showing that right-oriented individuals favour conformity more strongly than left-oriented individuals (e.g., Duriez, Luyten, Snauwaert, \& Hutsebaut, 2002; Jost et al., 2003). They are thus more likely to support status-quo and to rely on societal defaults, here in terms of global interpretations of perceptually ambiguous information.

We measured the relationship between perceptual bias and political attitude in two experiments. Perceptual bias was indexed using two tasks based on Navon's hierarchical figures (i.e., global shapes made of local elements; Navon, 1977; Fig 1a) and one on the Ebbinghaus illusion (De Fockert, Davidoff, Fagot, Parron, \& Goldstein, 2007; Fig 1c). In the 'Search Navon' task, participants had to discriminate a target represented either at the local or at the global level of the display (Derryberry \& Reed, 1998; Fig 1a). In the 'Similarity-matching Navon' task, participants made subjective preference choices about which of two comparison figures most resembled a target figure (Davidoff et al., 2008; Fig 1b). Finally, in the Ebbinghaus illusion, participants compared the size of two target circles surrounded by irrelevant inducers which affected their perceived size (De Fockert et al., 2007; Fig 1c). Political attitude was measured using the combination of three scales: an auto-evaluation scale (Jost et al., 2003), a political questionnaire developed by Cald (2011), and a questionnaire concerning a major political debate of direct relevance to participants in this study (i.e., a debate over university fees that took place in Québec in 2012).

In Experiment 2, we investigated the mediating role of cognitive rigidity in the relationship between political attitude and perceptual bias. Political studies have operationalised cognitive rigidity notably in terms of intolerance of ambiguity, intolerance of uncertainty and need for closure (Jost et al., 2003; Jost \& Amodio, 2012). We assessed these dimensions using adaptations of the ambiguity-intolerance questionnaire (Budner, 1962), uncertainty-intolerance questionnaire (Freeston, Rhéaume, Letarte, Dugas, \& Ladouceur, 1994) and Rigidity Breskin Test (Breskin, 1968) which assesses one's need for perceptual closure.

If right-oriented individuals indeed show a stronger global bias than left-oriented individuals, they should be less able to ignore irrelevant (distracting) contextual information in the Ebbinghaus illusion and they should be more consistently biased into interpreting ambiguous Navon figures in terms of their global shape. We expect that cognitive rigidity should play an important role in explaining these effects.

\section{Experiment 1}

\subsection{Method}

\subsubsection{Participants}

Sixty-five French-speaking participants ( 29 females, mean age 23 years, $S D=4.5$, age range 19-47 years), recruited from the Université du Québec à Trois-Rivières, in the province of Québec, Canada, participated in the study. The first author has previously used a similaritymatching Navon task to test individual differences in perceptual bias (Caparos et al., 2012, and other data in preparation for publication) and has found effects of medium size. With 
correlational designs, 65 participants are necessary to detect medium-sized effects ( $r$ values between .25 and .30; Draper \& Smith, 1998). On this basis, the number of participants was predetermined and testing stopped when the sample was complete. One participant did not complete all the tasks and was thus excluded from the analyses. Participants were compensated 10 Canadian dollars for participating.

\subsubsection{Stimuli and procedure}

Participants performed three computer-based perceptual tasks (in a counterbalanced order), after which they answered a paper-based questionnaire measuring their political opinions. The experimental tasks were presented using E-Prime 2.10 (Schneider, Eschman, \& Zuccolotto, 2002) on a 22-in LCD screen at a viewing distance of $60 \mathrm{~cm}$.

\subsubsection{Search Navon task}

A hierarchical Navon-like figure (Navon, 1977; Fig 1a) was presented on each trial, appearing equally often at the centre of the screen, $1.8^{\circ}$ of visual angle above the centre of the screen, or $1.8^{\circ}$ of visual angle below the centre of the screen. In each Navon figure, local elements (squares, crosses, triangles or circles; subtending $0.8^{\circ}$ ) were spaced equally apart from each other and were arranged to create a larger shape - the global shape (square, cross, triangle or circle; subtending $6.0^{\circ}$; Fig 1a). The different Navon figures occurred equally often across 96 trials presented in an intermixed and random order.

At the beginning of each trial, a fixation circle (subtending $0.5^{\circ}$ ) appeared at the centre of the screen for $1000 \mathrm{~ms}$, followed by a blank screen for $500 \mathrm{~ms}$. One of the Navon figures was then presented for $250 \mathrm{~ms}$, after which it was replaced by a blank screen until a response was recorded. Participants were instructed to find which of two targets, a cross or a square, was represented in the Navon figure. They pressed the ' $x$ ' key (on the computer keyboard) to respond 'cross' and the ' $m$ ' key to respond 'square'. The target was represented either at the global level, in the global-target condition, or at the local level, in the local-target condition. Participants were instructed to respond as quickly and accurately as possible.

\subsubsection{Similarity-matching Navon task}

Each display consisted of one target figure, presented at the top of the display, and two comparison figures, presented at the bottom of the display (Fig 1b). The three figures were at equal distance from each other ( $4.2^{\circ}$ away from the centre of the display). Each figure was a hierarchical Navon-like figure (Navon, 1977), in which local circles, squares or crosses (subtending $0.5^{\circ}$ ) - the local elements - were spaced equally apart from each other and were arranged to create a larger circle, square or cross (subtending $3.0^{\circ}$ ) - the global shape.

The task consisted in indicating which of the two comparison figures "looks most like" the target figure by pressing the left key (' $x$ ') or right key (' $m$ ') on the computer keyboard, respectively for the left or right comparison figure. On three control displays, there was a 
correct response: one comparison figure shared both (global and local) levels of similarity with the target (i.e., it was identical to the target) while the other figure shared no level of similarity with the target. All participants were $100 \%$ accurate on those displays. On nine test displays, there was no objectively correct response: each comparison figure shared either global or local similarity with the target (randomly presented to the left or right). When participants chose the comparison figure sharing global similarity, they were said to have made a 'global match'. Each display was presented until a response was given. Control and test displays were presented in an intermixed and random order.

\section{1a. Search Navon task}

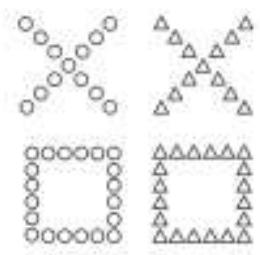

Global targets
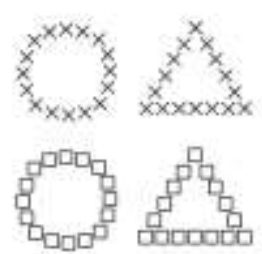

Local targets 1b. Similarity-matching Navon task

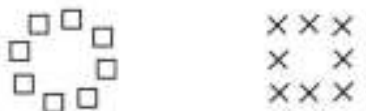

1c. Ebbinghaus illusion task

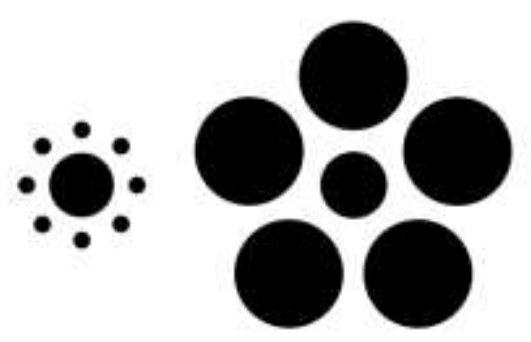

1d. Breskin Rigidity Task

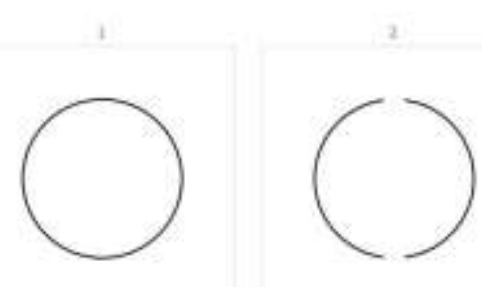

Figure 1. The figure represents (1a) the four global targets and the four local targets used in the search Navon task; (1b) one of the test displays used in the similarity-matching Navon task, containing one target figure (top figure) and two comparison figures (bottom figures); (1c) one of the test displays used in the Ebbinghaus illusion, containing two target circles surrounded, respectively, by small and large inducers (in this example, while the small-inducer target appears to be the biggest, in reality the large-inducer target is the biggest); and(1d) one of the 13 items used in the Breskin Rigidity Test, with one 'good Gestalt' shape (left) and one 'broken Gestalt' shape (right).

Because the strength of local vs. global percept can vary according to characteristics of the stimuli (e.g., denser Navon figures can increase global bias; Caparos, Linnell, Bremner, De Fockert, \& Davidoff, 2013), the amount of global matches varies from one display to another. The task was constructed by choosing three 'strongly-global' displays (yielding more than $85 \%$ global matches in the British-participant data of Caparos et al., 2012), three 'moderately global' 
displays (yielding between 80 and $85 \%$ global matches), and three 'weakly global' displays (yielding less than $80 \%$ global matches). According to the ambiguity-tolerance hypothesis, individual differences should be strongest with the most ambiguous 'weakly-global' displays.

\subsubsection{Ebbinghaus illusion task}

On each trial, two target circles were presented along the horizontal midline of the display, at equal distance ( $4.2^{\circ}$ of visual angle) from its centre (Fig 1c). One target, always measuring $2.0^{\circ}$ in diameter, was surrounded by small inducers (each measuring $0.5^{\circ}$ ). The other target, measuring from $1.86^{\circ}$ to $2.35^{\circ}$ (in $0.07^{\circ}$ steps), was surrounded by large inducers (each measuring $\left.3.35^{\circ}\right)$. The two targets occurred equally often on each side of the display. There were two conditions where the large-inducer target was smaller than the small-inducer target, one condition where both targets had the same size, and five conditions where the largeinducer target was larger than the small-inducer target. This asymmetry of target differences in the stimulus set was implemented to avoid a large number of redundant conditions (large inducers never produce the illusion of a larger target), and also meant that the median condition in the range did not present veridically equal targets.

Blocks of eight practice trials were first administered where the two targets were not surrounded by inducers. Participants decided which target was the largest by pressing the left $\left({ }^{\prime} x\right.$ ') or right (' $m$ ') key on the computer keyboard. The display remained until a response was given. After training, participants were presented with a block of 48 test trials (six trials for each target-size configuration).

\subsubsection{Political questionnaire}

In order to measure participants' political attitude, three scales were combined (e.g., Dodd et al., 2012). The first scale was a self-evaluation measure on which participants reported their ideological position, from 1 (politically to the left) to 5 (politically to the right).

The second scale evaluated participants' opinion a political debate over increasing university fees (the 2012 'maple spring'). Two questions were used (each with five response choices, from left-oriented to right-oriented): one regarding participants' opinion on the issue (in favour of: total gratuity/frozen fees/no opinion/fees indexed to prices/increase in fees), and the other regarding participants' involvement in demonstrations, where a red square was worn by opponents to the fee increase and a green square by those in favour (wore red square/sympathetic with red square/indifferent /sympathetic with green square/ wore green square).

Third, a twelve-item French-language scale developed by Cald (2011) assessed participants' opinions related to three major political axes, namely, (i) a socio-economic axis, (ii) an identity and responsibility axis, and (iii) a moral values axis. For each item of the scale, different policies were proposed and participants chose the policy they most agreed with. Each 
Political attitude and perceptual bias

policy was associated with a mark from 1 (left) to 5 (right). The overall score for the third scale was obtained by averaging the mean scores of the three axes.

The scores to the three scales were weighted equally and averaged to create a broad measure of left-right political attitude, ranging from 1 (politically to the left) to 5 (politically to the right).

\subsection{Results}

\subsubsection{Political attitude}

First, a reliability analysis (in which we entered the scores for the first two scales and the scores for the three axes of the third scale) produced a Cronbach's alpha of .75, confirming that the scale was reliable. The group mean political scores was 2.21 (range=1.06-3.85; median=2.11; $S D=0.60)$, which fell to the left of the political centre (3.0), $t(63)=9.86, p<.001, d=1.77$. Such finding was expected, both because participants were university students (a left-oriented population; American Freshman: National Norms Fall 2012, http://www.heri.ucla.edu) and were from Québec (where left-oriented ideology dominates; Ornstein, Stevenson, \& Stevenson, 2003). While not ideal, left biases in samples of political studies are common (Amodio et al., 2007; Kanai et al., 2011) and should not diminish the generalizability of the findings.

\subsubsection{Search Navon task}

For each participant, we calculated mean RT (for accurate responses) for local- and global-target conditions. Participants were faster in the global-target condition ( $M=328.8 \mathrm{~ms}$, $S D=97.5)$ than in the local-target condition ( $M=353.6 \mathrm{~ms}, S D=97.5), t(65)=3.6, p<.001, d=0.27$, $\mathrm{Cl}$ 95\% [11.0, 38.7], replicating previous findings of a global bias in Western participants (e.g., Caparos et al., 2012). The difference between local- and global-target RTs was computed for each participant and was used as an index of global bias (where higher values indicate more pronounced global bias). There was a positive relationship, of medium size, between political attitude and global bias, $r(64)=.25, p=.047, \mathrm{Cl} 95 \%[.01, .47]$ : right-oriented participants showed stronger global bias (Fig 2a).

\subsubsection{Similarity-matching Navon task}

Percentages of global and local matches were first computed; across all display types, participants mostly made global matches ( $M=79 \%, S D=24)$, similarly to what is typically observed in western samples (Caparos et al., 2012; Davidoff et al., 2008). The relationship between political score and global matches was reliable for weakly-global displays, $r(64)=.28, p$ $=.025, \mathrm{Cl} 95 \%[.04, .49]$ but not for strongly- or moderately-global displays, respectively, $r(64)=$ $-.02, p=.896, \mathrm{Cl} 95 \%[-.26, .23]$, and $r(64)=.02, p=.905, \mathrm{Cl} 95 \%[-.23, .26]$. This finding indicated a stronger global bias in right-oriented participants (Fig $2 b$ ) for displays that were most ambiguous (i.e., less likely to be processed globally). 

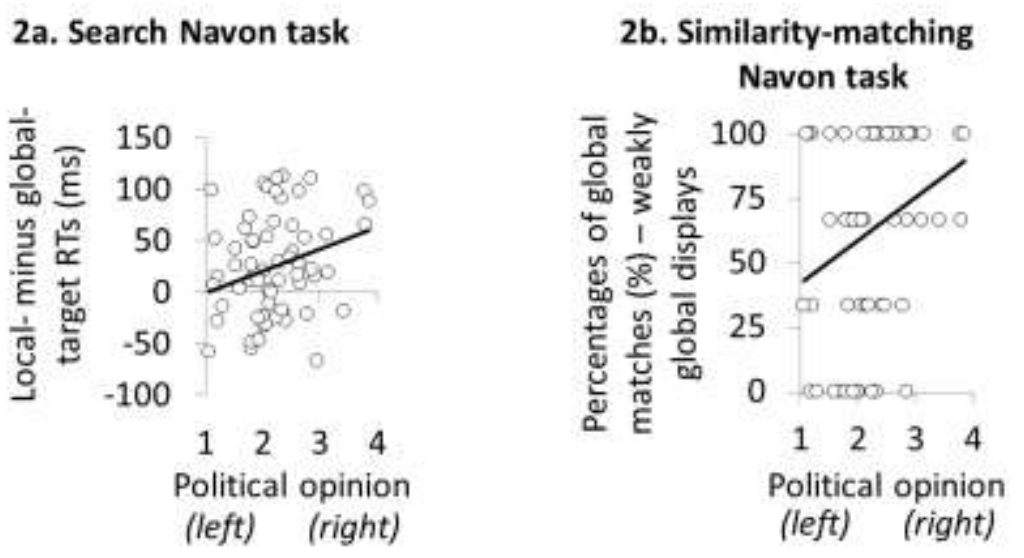

2c. Ebbinghaus illusion task

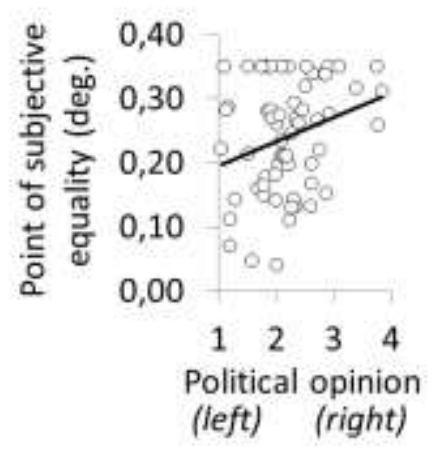

Figure 2. Experiment 1. Relationship between political attitude and (2a) local- minus globaltarget RTs (ms) in the search Navon task, (2b) global matches with weakly global displays in the similarity-matching Navon task, and (2c) point of subjective equality in the Ebbinghaus illusion.

\subsubsection{Ebbinghaus illusion task}

Three participants who had chosen the small-inducer target $100 \%$ of the time were excluded from the analysis. For each remaining participant we computed the point of subjective equality (PSE), that is, the threshold (in terms of size difference between large- and smallinducer targets) where the two targets appeared to have the same size. Larger PSEs indicate a more pronounced effect of contextual information, a stronger illusion. We fitted the data with the model: $p=\phi([k-d] / \sigma$, where $p$ is the probability of choosing the target with large inducers, $\phi(z)$ is the inverse cumulative distribution function for a standard normal distribution, $k$ is the required threshold for deciding that the target with large inducers is the larger one, $d$ is the difference between the radius of the two circles (in degrees of visual angle), and $\sigma$ is the standard deviation of the normally distributed noise from all sources.

Participants' mean PSE ( $\left.\mathrm{M}=0.24^{\circ}, \mathrm{SD}=.09\right)$ was similar to that observed previously in a western population (Caparos et al., 2012). There was a positive relationship, of medium size, between political attitude and PSE, $r(61)=.28, p=.032, \mathrm{Cl} 95 \%[.03, .50]$ : the strength of the 
visual illusion was stronger in right-oriented participants, suggesting a greater sensitivity to contextual information (Fig 2c).

\section{$\underline{2.2 .5 \text { Discussion of results }}$}

Consistent with the cognitive-rigidity hypothesis, results of Experiment 1 showed that right-oriented participants had a stronger perceptual bias. This was observed across three different measures. Though consistent with our prediction that was derived from the premise of differences in cognitive rigidity, evidence of the implication of this construct remains indirect as it was not measured in Experiment 1.

In Experiment 2, the mediating role of cognitive rigidity was tested directly. In addition, we investigated the role of a number of other factors which could be related to political attitude and could account for the relationship between political attitude and perception. Specifically, we investigated the role of rurality (Kerr, 1952), religiosity (Duriez et al., 2002), paranormal belief (which strongly correlates with religiosity; Orenstein, 2002), availability of financial resources (Cohen, Vigoda, \& Samorly, 2001), media exposure (McLeod \& McDonald, 1985), multilingualism and diversity of cultural heritage (Xu, Mar, \& Peterson, 2013). Rurality has been shown to affect perceptual bias (Caparos et al., 2012). All of the other factors listed might affect holistic thinking, and thus global perception (Nisbett, Peng, Choi, \& Norenzayan, 2001). Because all these factors are linked to political attitude, they could indirectly explain the relationship between political attitude and perceptual bias.

\section{Experiment 2}

\subsection{Method}

\subsubsection{Participants}

Eighty-seven French-speaking participants completed an online questionnaire. The data of three participants were excluded from the analyses as they did not show $100 \%$ accuracy at the catch trials (see stimuli and procedure). The remaining 84 participants consisted of 52 females and 32 males, mean age 31.3 years ( $S D=10.5$, range 19-71 years).

\subsubsection{Stimuli and procedure (online questionnaire)}

The different questions of the online questionnaire were always presented in the same order. In a first section, participants entered demographic information (gender, age, country of birth, country of residence) and then answered a number of multiple-choice questions about their personal situation, namely, their immigration status ("citizen", "permanent resident", or "temporary resident"), the number of grand-parents born in a country other than their own (“0”, "1", "2", “3”, or "4"), their first language ("French", " English", "Spanish", or "Other"), the number of languages they speak fluently ("1", “2", “3", or "more than 3 "), the average population of their city of residence during the past 5 years ("less than 1 thousand", "between 1 and 10 thousand", "between 10 and 100 thousand", "between 100 thousand and 1 million", or 
"more than 1 million"), the average population of their city of residence during their first 15 years of life (same choices as preceding), and their financial resources ("month ends are difficult", "month ends are sometimes difficult", "I have what I need but am nevertheless careful" , "I am at ease financially", or "I am very much at ease financially").

In the second section of the online questionnaire, participants performed the 13-item adaptation of the Breskin Rigidity Test described by Maltby and Lewis (1996). Each item consisted of two geometrical shapes. Participants indicated which of two shapes was more aesthetically pleasing. One of the two shapes (the 'good Gestalt' shape) was a better Gestalt than the other shape (the 'broken Gestalt' shape). For instance, in the fifth item, one shape was a full circle (good Gestalt) and the other one was an incomplete circle (broken Gestalt; see Figure 1d). Each 'good Gestalt' choice was scored one. For each participant, the scores for each question were added, yielding an overall score that ranged from 0 to 13 . High overall scores indicated high cognitive rigidity (Breskin, 1968).

In the third section of the questionnaire, participants performed a 10-trial adaptation of the Ebbinghaus task used in Experiment 1 (see Method section of Experiment 1). Each trial consisted of two target circles, one of which was surrounded by small inducers, the other one by large inducers. Participants chose which target was larger by ticking one of two choices, namely, 'left central circle is larger' or 'right central circle is larger'. Because it is impossible to control for screen dimensions and distance of presentation with the mode of testing used in Experiment 2, we express stimulus sizes in percentage of the size of the small-inducer target (i.e., the reference target, which had a constant size across trials). The large-inducer target measured, from trial 1 to 10 respectively, 126, 91, 106, 126, 106, 112, 91, 112, 118, and $118 \%$ of the size of the small-inducer target. The large-inducer target occurred as often to the left as to the right of the small-inducer target, in a pseudo-random order. Inducers measured, respectively, $26 \%$ (small inducers) and 149\% (large inducers) of the size of the small-inducer target. On trials 2 and 7 , the small-inducer target was the largest, thus in those trials the correct response was reinforced by the illusion and participants were expected to give an accurate response. These two trials were used as catch trials (i.e., to ensure that participants were on task). On trials 1 and 4, the large-inducer target was markedly larger than the small-inducer target, thus in those trials participants were expected to give an accurate response despite the effect of the illusion. These two trials were used as control trials (see Results section below). For each participant, the strength of the illusion was obtained by averaging the accuracy for the remaining 6 test trials (because few trials were used in Experiment 2, it was not possible to extract the point of subjective equality as in Experiment 1).

In the fourth section of the questionnaire, participants performed a 4-trial adaptation of the similarity-matching Navon task used in Experiment 1 (see Method section of Experiment 1). The task consisted in indicating which of two comparison figures "looks most like" the target figure by ticking one of two choices, namely, "the left figure looks most like the top figure" or "the right figure looks most like the top figure". The first trial used one control display (see Experiment 1), where one of the comparison figures matched the target figure both at the local 
Political attitude and perceptual bias

and at the global level. This trial was used as a catch trial (i.e., to ensure that participants were on task). Trials 2 to 4 used the three 'weakly-global' test displays from Experiment 1. Mean percentage of global matches across those three test trials was used as an estimate of perceptual bias.

In the fifth section of the questionnaire, participants filled the three scales used in Experiment 1 to measure political attitude (see Method section of Experiment 1). The moralvalue axis was removed from the third scale (i.e., from the Cald questionnaire) given that, in Experiment 1, this scale correlated weakly with the other scales. Participants also filled an 8item French adaptation of Budner's (1962) 'intolerance of ambiguity' scale (e.g., "People who want clear 'yes-no' answers do not imagine the complexity of things; I agree; I somewhat agree; I neither agree nor disagree; I somewhat disagree; I disagree"), and a 5-item adaptation of Freeston et al.'s (1994) 'intolerance of uncertainty' scale (e.g., “Uncertainty makes me uneasy, anxious or stressed; I agree; I somewhat agree; I neither agree nor disagree; I somewhat disagree; I disagree"). In both scales, each question was scored from 1 (low ambiguity/ uncertainty intolerance) to 5 (high ambiguity/uncertainty intolerance). A mean score (ranging from 1 to 5) was obtained for each scale by averaging the scores to each question.

Finally, participants answered multiple-choice questions, about (1) paranormal belief ("I do not believe at all in paranormal activity", "I believe that it is unlikely that paranormal activity exists", "I believe it is possible that paranormal activity exists", or "I am convinced in the existence of paranormal activity"), (2) source of media exposure ("I don't consult the news", "I consult the news mainly via my e-mail provider, e.g., yahoo news", "I consult the news on radio or television", or "I visit dedicated news websites, e.g., LaPresse.ca, LeMonde.fr"), (3) frequency of media exposure ("I almost never consult the news", "I consult the news less than once a week", "I consult the news several times a week", "I consult the news every day", or "I consult the news several times a day"), and (4) religious practice ("I am an atheist", "I am not sure about the existence of god", "I believe in god but I am not a follower; I do not visit places of worship", "I believe in god and I am a follower; I sometimes visit places of worship", or "I believe in god and I am a strong follower; I often visit places of worship").

\subsection{Results}

\subsubsection{Cognitive rigidity}

The group mean ambiguity-intolerance score was 2.59 (range=1.60-4.30; median=2.56; $\mathrm{SD}=0.48$ ); the group mean uncertainty-intolerance score was 2.43 (range=1.00-4.40; median=2.40; $S D=0.69$ ); and the group mean Breskin-rigidity score was 6.33 (range=1.00-12.00; median=6.00; $\mathrm{SD}=3.08$ ).

There was a positive relationship between ambiguity-intolerance score and uncertaintyintolerance score, $r(84)=.34, p=.001, \mathrm{Cl} 95 \%[-.06, .36]$, and a non-significant trend for a positive relationship between ambiguity-intolerance score and Breskin-rigidity score, $r(84)=.18$, 
$p=.098, \mathrm{Cl} 95 \%[-.06, .36]$. There was no relationship between uncertainty-intolerance and the Breskin-rigidity scores, $r(84)=.05, p=.669, \mathrm{Cl} 95 \%[-.06, .36]$.

\subsubsection{Political attitude}

A reliability analysis (in which we entered the scores for the first two political scales and the scores for the two axes of the third political scale) produced a Cronbach's alpha of .82, confirming that the political questionnaire was reliable. The group mean political score was 2.39 (range=1.00-4.52; median=2.19; $S D=0.93$ ), which fell to the left of the political centre (3.0), $t(83)$ $=5.98, p<.001, \mathrm{~d}=0.968$, like in Experiment $1 .{ }^{1}$

Political score was positively related (1) to ambiguity-intolerance score, $r(84)=.45, p<$ $.001, \mathrm{Cl} 95 \%[.26, .60]$ (Fig 3c) and (2) to uncertainty-intolerance score, $r(84)=.28, p=.010, \mathrm{Cl}$ $95 \%[.07, .47]$. There was also a non-significant trend for a positive relationship between political score and Breskin Rigidity score, $r(84)=.16, p=.134, \mathrm{Cl} 95 \%[-.06, .36]$.

Political score was related to a number of the control variables we measured. It was positively related to religiosity score (see Table 1 ) and negatively related (1) to population of childhood city of residence, (2) to population of current city of residence, and (3) to number of languages spoken fluently. There was also a non-significant trend for a negative relationship between political score and number of grandparents born abroad. There was no other relationship between political score and the control variables (see Table 1). In addition, political score was similar across males and females, $t(82)=0.23, p=.817$.

In sum, compared to left-oriented participants, right-oriented participants were more intolerant of ambiguity and uncertainty, more religious, they spoke fewer languages, and they grew up and currently lived in smaller cities. They might also have been more rigid at the Breskin Rigidity Test.

\subsubsection{Similarity-matching Navon task}

In the similarity-matching Navon task, like in Experiment 1, there was a medium-size positive relationship between political score and global matches, $r(84)=.27, p=.012, \mathrm{Cl} 95 \%$ $[.06, .46]$ (Fig 3a). This finding confirmed the presence of a stronger global bias in right-oriented participants.

There were also positive relationships (1) between ambiguity-intolerance score and global matches, $r(84)=.23, p=.038, \mathrm{Cl} 95 \%[.02, .42]$ (Fig 3d), and (2) between Breskin Rigidity score and global matches, $r(84)=.23, p=.034, \mathrm{Cl} 95 \%[.02, .42]$. However, the relationship

\footnotetext{
${ }^{1}$ Fifteen non-Quebecer participants did not have an opinion about the University Fee debate (i.e., they had a missing value for the second political scale; see Method section of Experiment 1). For these participants, political score was calculated by averaging first- and third-scale scores. The analyses described in the Results section were also performed by excluding those 15 participants and the outcomes were similar.
} 
between uncertainty-intolerance score and global matches was not significant, $r(84)=.04, p=$ $.743, \mathrm{Cl} 95 \%[-.18, .25]$. In addition, none of the control variables tested in Experiment 2 was related to percentage of global matches (see Table 1 ).

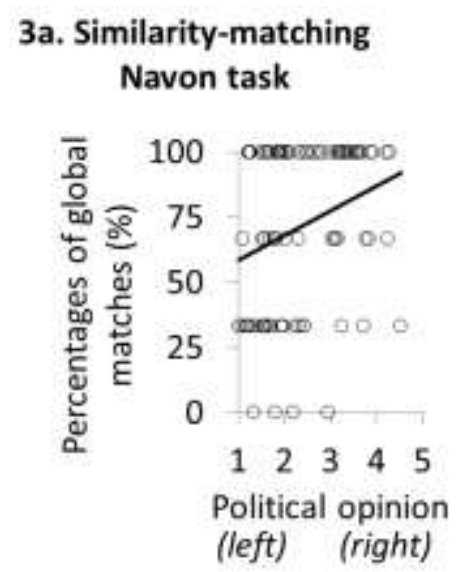

\section{3b. Ebbinghaus illusion task}

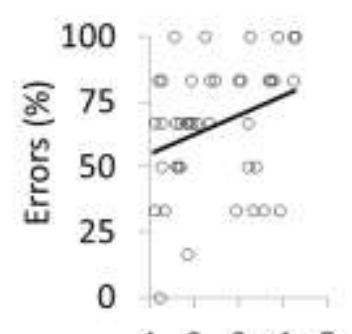

$\begin{array}{lllll}1 & 2 & 3 & 4 & 5\end{array}$ Political opinion (left) (right) 3c. Ambiguity intolerance
questionnaire

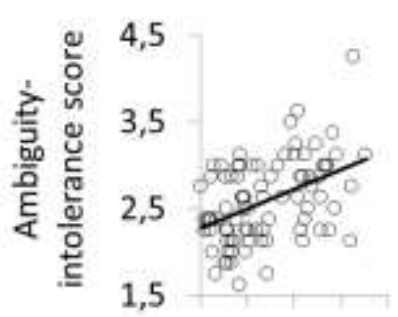

$\begin{array}{llllll}1 & 2 & 3 & 4 & 5\end{array}$

Political opinion (left) (right)
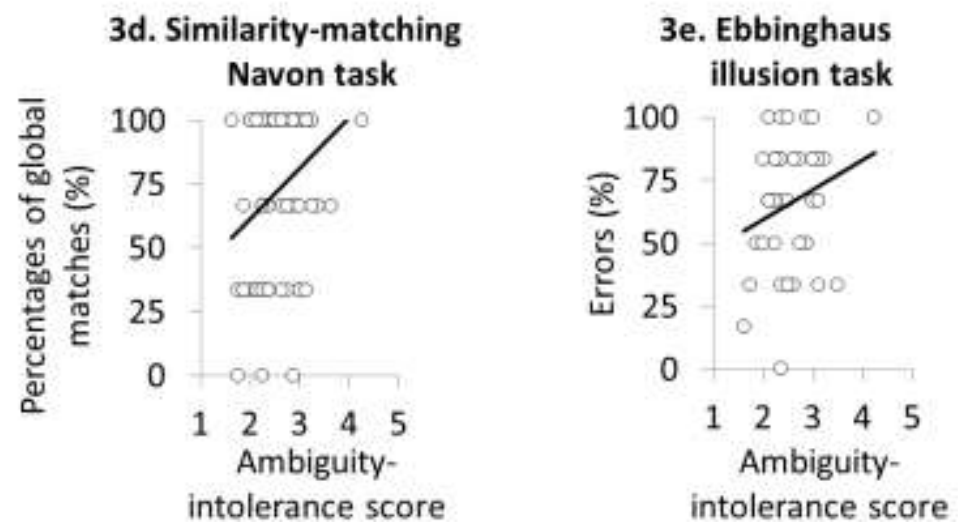

Figure 3. Experiment 2. Relationship between political attitude and (3a) global matches in the similarity-matching Navon task, (3b) error percentages in the Ebbinghaus illusion and (3c) ambiguity-intolerance score. Relationship between ambiguity-intolerance score and (3d) global matches in the similarity-matching Navon task and (3e) error percentages in the Ebbinghaus illusion.

When ambiguity-intolerance score and Breskin Rigidity score were entered as control variables in a partial correlation, the relationship between political score and global matches became non-significant, $r(80)=.18, p=.099, \mathrm{Cl} 95 \%[-.04, .38]$. When we controlled for the other variables shown to be related to political attitude (religiosity, multilingualism, childhoodcity population and current-city population), the relationship between political score and global matches remained significant, $r(78)=.23, p=.042, \mathrm{Cl} 95 \%[.02, .45]$.

In sum, intolerance of ambiguity and rigidity at the Breskin Test played a mediating role in the relationship between political attitude and global matches. In contrast, the control 
variables tested in Experiment 2 did not explain the relationship between political attitude and global matches.

\subsubsection{Ebbinghaus illusion}

When we analysed the data of all 84 participants, we did not find a significant relationship between political score and Ebbinghaus illusion size, $r(84)=.15, p=.179, \mathrm{Cl} 95 \%[-$ $.07, .35]$. This might have been because half of the participants had consistently chosen the small-inducer target, even in the two control trials (where the large-inducer target was markedly larger than the small-inducer target).

In a second analysis, we selected only the 41 participants who had given a correct response to both control trials. In this subset of participants, like in Experiment 1, there was a medium-size positive relationship between political score and illusion size, $r(41)=.31, p=.053$, $\mathrm{Cl} 95 \%[.00, .56]$ (Fig 3b). This finding confirmed a stronger global bias in right-oriented participants.

There was also a positive relationship between uncertainty-intolerance score and illusion size, $r(41)=.33, p=.038, \mathrm{Cl} 95 \%[.02, .58]$, and a non-significant trend for a positive relationship between ambiguity-intolerance score and illusion size, $r(41)=.25, p=.123, \mathrm{Cl} 95 \%$ $[-.07, .51]$ (Fig 3e). On the other hand, there was no relationship between Breskin Rigidity score and illusion size, $r(41)=.08, p=.645, \mathrm{Cl} 95 \%[-.24, .37]$. There was no significant relationship between the control variables and illusion size (but there were some non-significant trends; see Table 1)

Table 1. Pearson's correlation coefficients for control variables (religiosity, current-city population size, childhood-city population size, multilingualism, diversity of cultural heritage, availability of financial resources, media exposure, and paranormal belief) with political attitude, global matches at the similarity-matching Navon task, and Ebbinghaus illusion size.

\begin{tabular}{cccc}
\hline & $\begin{array}{c}\text { Political attitude } \\
(\mathrm{N}=84)\end{array}$ & $\begin{array}{c}\text { Global matches } \\
(\mathrm{N}=84)\end{array}$ & $\begin{array}{c}\text { Illusion size } \\
(\mathrm{N}=41)\end{array}$ \\
\hline Religiosity & $.354^{* *}$ & .118 & .102 \\
Current-city pop. & $-.413^{* *}$ & -.148 & $.297^{\dagger}$ \\
Childhood-city pop. & $-.291^{* *}$ & -.052 & .254 \\
Multilingualism & $-.232^{*}$ & -.046 & -.218 \\
Cultural diversity & $-.182^{\dagger}$ & -.128 & $-.297^{\dagger}$ \\
Financial resources & .016 & -.018 & $.267^{\dagger}$ \\
Media exposure & .086 & .040 & .216 \\
Paranormal belief & .129 & .111 & -.150 \\
Age & -.160 & .018 & -.027 \\
\hline
\end{tabular}

Note: ${ }^{\dagger} p<.100,{ }^{*} p<.050,{ }^{* *} p<.010$ 
When ambiguity-intolerance score and uncertainty-intolerance score were entered as control variables in a partial correlation, the relationship between political score and illusion size became non-significant, $r(37)=.170, p=.300, \mathrm{Cl} 95 \%[-.16, .47]$. When we controlled for the other variables shown to be related to political attitude or to illusion size (religiosity, multilingualism, childhood-city population, current-city population, and financial resources), the strength of the relationship between political score and illusion size did not decrease, $r(33)=$ $.381, p=.024, \mathrm{Cl} 95 \%[.04, .64]$ (if anything, it increased).

In sum, intolerance of ambiguity and intolerance of uncertainty played a mediating role in the relationship between political attitude and the size of the Ebbinghaus illusion. In contrast, the control variables tested in Experiment 2 did not explain the relationship between political attitude and illusion size.

\subsubsection{Discussion of results}

Experiment 2 replicated the positive relationship between political attitude and perceptual bias. It also showed evidence that this relationship is mediated by cognitive rigidity. Specifically, (1) the relationship between political attitude and global matches in the similaritymatching Navon task was mediated by intolerance of ambiguity and rigidity at the Breskin Test, and (2) the relationship between political attitude and Ebbinghaus illusion size was mediated by intolerance of ambiguity and intolerance of uncertainty. The other control variables measured in Experiment 2 (rurality, religiosity, paranormal belief, multilingualism, diversity of cultural heritage, availability of financial resources, or media exposure) did not account for the relationship between political attitude and perceptual bias.

\section{General Discussion}

The present study provides evidence for a link between political attitude, a high-level construct, and low-level perceptual processes. Compared to left-oriented individuals, rightoriented individuals are more influenced by contextual information, in the Ebbinghaus illusion, and they more readily perceive geometrical figures in terms of their global shape, even when these figures do not strongly encourage a global interpretation. Effects were of medium size and consistent across tasks and experiments (Fig 4). The relationship between political attitude and perceptual bias was mediated by cognitive rigidity. 


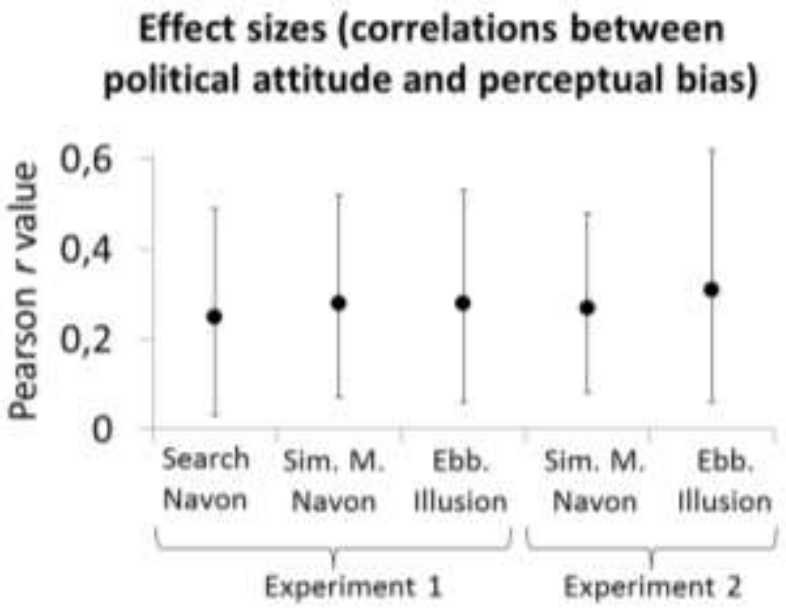

Figure 4. Pearson r values across perceptual tasks (search Navon, similarity-matching Navon, or Ebbinghaus illusion), in Experiments 1 and 2 (error bars represent 95\% confidence interval).

The directionality of the link between political attitude and perception may be conceived as top-down, where the thinking style of right-oriented individuals - characterised by higher rigidity (Jost et al., 2003) - increases the tendency to always resolve perceptual ambiguity in the same way. According to this view, while right-oriented ideology should be associated with stronger perceptual bias in all cultures, the direction of this increase in bias might vary across cultures. While right-oriented ideology would promote strong global perception in western cultures where global perception is prominent, it might promote stronger local perception in cultures where local perception is prominent (e.g., remote traditional cultures; Davidoff et al., 2008; Hypothesis A). This view is consistent with a finding showing that remote people (the Himba, in Northern Namibia) who visited a local town less often, and may therefore be less open to novelty and more conservative, presented a stronger local bias than their peers who visited the local town more often (Caparos et al., 2012). Irrespective of culture, left-oriented ideology would be associated with less biased perception (i.e., where local and global interpretations are equally prominent; Hypothesis A).

An alternative bottom-up account of the findings might hold that perception guides our conceptualisation of the world (Barsalou, 1999; Förster, 2009) and, as a result, influences our cognitive rigidity and shapes our belief system and political attitudes. More local perception might emphasize the salience of odd patterns and rare cases, which would increase their cognitive importance. This might promote a more flexible, diversity-oriented ideology, which would be generally more compatible with left-oriented political values. According to this account, while strongly global perception would favour right-oriented ideology, strongly local perception would favour left-oriented ideology. One would thus predict that, even in remote cultures, strongly local perception should be associated with more left-oriented (liberal) views (Hypothesis B). 
Political attitude and perceptual bias

The present study does not allow causal conclusions on this specific issue. Future work might investigate causal effects more directly using experimental manipulations to determine whether (1) the priming of local or global perception (e.g., Förster, 2009; Gao, Flevaris, Robertson, \& Bentin, 2011) influences participants' political views or (2) whether the priming of liberal or conservative views (e.g., Nail, McGregor, Drinkwater, Steele, \& Thompson; 2009) affects perceptual bias.

Perceptual bias, once believed to be generally fixed and shared across humankind (Navon, 1977), has been shown to be plastic and sensitive to a number of factors, including culture (Doherty, Tsuji, \& Phillips, 2008) and urbanisation (Caparos et al., 2012). For the first time, this study offers evidence of a link between political attitudes, cognitive rigidity and perceptual bias. Our work adds to recent findings showing that political attitudes are traceable to early and basic cognitive mechanisms. It shows that right- and left-oriented individuals actually see the world differently.

\section{References}

Amodio, D. M., Jost, J. T., Master, S. L., \& Yee, C. M. (2007). Neurocognitive correlates of liberalism and conservatism. Nature Neuroscience, 10, 1246-1247. DOI: 10.1038/nn1979

Amodio, D. M., \& Jost, J. T. (2012). Political ideology as motivated social cognition: Behavioral and neuroscientific evidence. Motivation and Emotion, 36, 55-64. DOI: 10.1037/0033-

2909.129.3.339

Barsalou, L. W. (1999). Perceptions of perceptual symbols. Behavioral and Brain Sciences, 22, 637-660. DOI: 10.1017/S0140525X99532147

Budner, S. (1962). Intolerance of ambiguity as a personality variable. Journal of Personality, 30, 29-50. DOI: 10.1111/j.1467-6494.1962.tb02303.x

Cald, L. (2011). Êtes-vous de gauche ou de droite? Pour révéler votre identité politique. Paris: Max Milo.

Caparos, S., Linnell, K. J., Bremner, A., De Fockert, J., \& Davidoff, J. (2013). Do local and global perceptual biases tell us anything about local and global selective attention? Psychological Science, 24, 206-212. DOI: 10.1177/0956797612452569

Caparos, S., Ahmed, L., Bremner, A., De Fockert, J., Linnell, K. J., \& Davidoff, J. (2012). Exposure to an urban environment alters the local bias of a remote culture. Cognition, 122, 80-85. DOI: 10.1016/j.cognition.2011.08.013

Cohen, A., Vigoda, E., \& Samorly, A. (2001). Analysis of the Mediating Effect of PersonalPsychological Variables on the Relationship Between Socioeconomic Status and Political Participation: A Structural Equations Framework. Political Psychology, 22, 727-757. DOI: 10.1111/0162-895X.00260 
Political attitude and perceptual bias

Davidoff, J., Fonteneau, E., \& Fagot, J. (2008). Local and global processing: observations from a remote culture. Cognition, 108, 702-709. DOI: 10.1016/j.cognition.2008.06.004

De Fockert, J., Davidoff, J., Fagot, J., Parron, C., \& Goldstein, J. (2007). More accurate size contrast judgments in the Ebbinghaus Illusion by a remote culture. Journal of Experimental Psychology: Human Perception and Performance, 33, 738-742. DOI: 10.1037/00961523.33.3.738

Derryberry, D., \& Reed, A. (1998). Anxiety and attentional focusing: trait, state and hemispheric influences. Personality and Individual Differences, 25, 745-761. DOI: 10.1016/S0191-

8869(98)00117-2

Doherty, M. J., Tsuji, H., \& Phillips, W. A. (2008). The context sensitivity of visual size perception varies across cultures. Perception, 37, 1426-1433. DOI: 10.1068/p5946

Dodd, M. D., Balzer, A., Jacobs, C. M., Gruszczynski, M. W., Smith, K. B., \& Hibbing, J. R. (2012). The political left rolls with the good and the political right confronts the bad: connecting physiology and cognition to preferences. Philosophical Transactions of the Royal Society: Biological Sciences, 367, 640-649. DOI: 10.1098/rstb.2011.0268

Draper, N. R., \& Smith, H. (1998). Applied Regression Analysis Wiley Series in Probability and Statistics. Wiley.

Duriez, B., Luyten, P., Snauwaert, B., \& Hutsebaut, D. (2002). The importance of religiosity and values in predicting political attitudes: Evidence for the continuing importance of religiosity in Flanders (Belgium). Mental Health, Religion \& Culture, 5, 35-54. DOI:

$10.1080 / 13674670110066831$

Förster, J. (2009). Relations between perceptual and conceptual scope: how global versus local processing fits a focus on similarity versus dissimilarity. Journal of Experimental Psychology: General, 138, 88-111. DOI: 10.1037/a0014484

Freeston, M. H., Rhéaume, J., Letarte, H., Dugas, M. J., \& Ladouceur, R. (1994). Why do people worry? Personality and individual differences, 17, 791-802. DOI: 10.1016/0191-8869(94)90048-5

Gao, Z., Flevaris, A. V., Robertson, L. C., \& Bentin, S. (2011). Priming global and local processing of composite faces: revisiting the processing-bias effect on face perception. Attention, Perception, \& Psychophysics, 73, 1477-1486. DOI: 10.3758/s13414-011-0109-7

Jost, J. T., Glaser, J., Kruglanski, A. W., \& Sulloway, F. J. (2003). Political conservatism as motivated social cognition. Psychological Bulletin, 129, 339. DOI: 10.1037/0033-2909.129.3.339

Kanai, R., Feilden, T., Firth, C., \& Rees, G. (2011). Political orientations are correlated with brain structure in young adults. Current Biology, 21, 677-680. DOI: 10.1016/j.cub.2011.03.017 
Political attitude and perceptual bias

Kerr, W. A. (1952). Untangling the liberalism-conservatism continuum. The Journal of Social Psychology, 35, 111-125. DOI: 10.1080/00224545.1952.9921835

Luguri, J. B., Napier, J. L., \& Dovidio, J. F. (2012). Reconstruing Intolerance: Abstract Thinking Reduces Conservatives' Prejudice Against Nonnormative Groups. Psychological Science, 23, 756763. DOI: $10.1177 / 0956797611433877$

McLeod, J. M., \& McDonald, D. G. (1985). Beyond simple exposure media orientations and their impact on political processes. Communication Research, 12, 3-33. DOI:

$10.1177 / 009365085012001001$

Ornstein, M., Stevenson, H. M., \& Stevenson, M. D. (2003). Politics and ideology in Canada: Elite and public opinion in the transformation of a welfare state. McGill-Queen's Press-MQUP.

Orenstein, A. (2002). Religion and paranormal belief. Journal for the Scientific Study of Religion, 41, 301-311. DOI: 10.1111/1468-5906.00118

Oxley, D. R., Smith, K. B., Alford, J. R., Hibbing, M. V., Miller, J. L., Scalora, M., Hatemi, P. K., \& Hibbing, J. R. (2008). Political attitudes vary with physiological traits. Science, 321, 1667-1670. DOI: $10.1126 /$ science. 1157627

Nail, P. R., McGregor, I., Drinkwater, A. E., Steele, G. M., \& Thompson, A. W. (2009). Threat causes liberals to think like conservatives. Journal of Experimental Social Psychology, 45, 901907. DOI: 10.1016/j.jesp.2009.04.013

Navon, D. (1977). Forest before trees: The precedence of global features in visual perception. Cognitive Psychology, 9, 353-383. DOI: 10.1016/0010-0285(77)90012-3

Nisbett, R. E., Peng, K., Choi, I., \& Norenzayan, A. (2001). Culture and systems of thought: holistic versus analytic cognition. Psychological Review, 108, 291-310. DOI: 10.1037/0033-

295X.108.2.291

Schneider, W., Eschman, A., \& Zuccolotto, A. (2002). E-Prime user's guide. Pittsburgh, PA: Psychology Software Tools.

Smith, K. B., Oxley, D. R., Hibbing, M. V., Alford, J. R., \& Hibbing, J. R. (2011). Linking genetics and political attitudes: Reconceptualizing political ideology. Political Psychology, 32, 369-397. DOI: 10.1111/j.1467-9221.2010.00821.x

Xu, X., Mar, R. A., \& Peterson, J. B. (2013). Does cultural exposure partially explain the association between personality and political orientation? Personality and Social Psychology Bulletin, 39, 1497-1517. DOI: 10.1111/0038-4941.00064

Yeung, N., Botvinick, M. M., \& Cohen, J. D. (2004). The neural basis of error detection: conflict monitoring and the error-related negativity. Psychological Review, 111, 931-959. 